\title{
Strategies on Prevention of Pollution from Rotary-molded Boat of the Marine Environment
}

\author{
Qiguo Yao 1, Le Xu 2
}

1 School of Naval Architecture \& Ocean Engineering, Zhejiang Ocean University, Zhoushan, Zhejiang, China.

yaoqiguo@163.com

2 School of Naval Architecture \& Ocean Engineering, Zhejiang Ocean University, Zhoushan, Zhejiang,China.

379417525@qq.com

Keywords: rotary-molded boat; pollution; marine environment; suggestion

\begin{abstract}
Due to the booming of world shipping industry, the pollution of marine environment from ships becomes more and more serious. Under the legal framework of International Maritime Organization (IMO), the MARPOL Convention made great contribution to the prevention pollution from ships. However, there are still some illegal discharging existing, hence, the reasons of these problems are analyzed from the ship owner, seafarers, legal affairs and surveillance of the administration aspects as follows. Furthermore, the possible hazards of marine oil pollution were discussed and related countermeasures were proposed, including implementation of laws and regulations, enhancing awareness of environment protection, improvement of pollution monitoring and early warning mechanism, physical treatment, chemical treatment and biological treatment. Meanwhile, some suggestions aimed at these problems are discussed in this paper.
\end{abstract}

\section{Introduction}

Ocean, abundant with resources, is material basis for development of marine economy and valuable for sustainable development of human society. With the world economy developing and globalization, ship still prevail over the cargos transportation in terms of weight, and it is about 90 percent of world total trade of goods measured in tons are moved by sea [1-3], meanwhile, the international trade drive the world fleet continuously increased, however, going with the shipping industry, various pollution from ships which significantly affect the environments, such as oil spill, air pollution, sewage discharge etc. Consequently, marine ecological environment and marine lives are endangered. It becomes urgent and necessary for marine environmental protection to be guaranteed. Furthermore, with the rapid development of coastal economic in China, many consumption projects will be carried out, such as leisure, vacation, maritime yachts etc. So, the rotary-molded boat market will grow much more, which is showed in Fig.1. How to cope with the pollution problems which troubles people a lot and the International Maritime Organization (IMO) do great contribution to the job of pollution prevention from all kinds of ships.

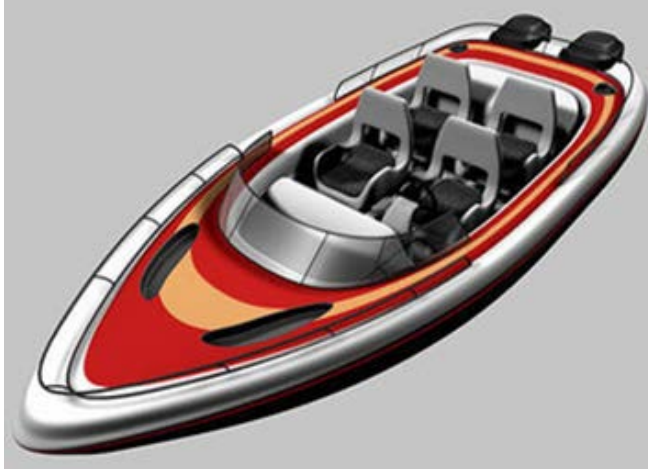

Fig.1 Contour diagram of a rotary-molded boat 


\section{Existing Problems}

The international convention MARPOL 73/78 is the specific convention for prevention pollution from ships, which provides operational standards, required equipments and some procedures etc [4]. Thereby, the kernel problem is that the implementation of the owners, crew members and the supervision of the administration. Unfortunately, there are problems existing here, for instance, from informal channels, it is known that many cases of using "magic pipes" to discharge oil water deliberately, why things happened like that? What can we do to diminish this phenomenon? There are some relevant factors and suggestions as follows.

\section{Pollution Source}

\section{Type of Pollution}

Marine pollution by input oil can be divided into sudden input and chronic input. The former involves tanker accidents, oil spill of offshore exploration and oil mining blow out accident and the latter contains oily wastewater emissions from port, ship or offshore areas, leakage of natural seabed, oil-bearing sedimentary rocks by sea erosion/exudation, discharge of industrial and residential wastewater, and subsidence of oily exhaust [5-7].

The cause of marine contamination incorporates that it is frequent maritime transport of petroleum and oil port-handling that matter in increase of oil spill accident, and hidden issues should be further explored. What's more, large tankers actually enhance the possibility of oil spills and improve difficulty of handling. In addition, leakage and discharge of waste water would occur in oil exploration and development in offshore fields.

\section{Existence of Marine Oil Pollution}

In general, the existence form of oil in ocean includes floating oil film, seawater in dissolved/dispersed state(including solution and emulsion state) and condensed state (including floating tar ball and residues in the sediments).

When discharged into the ocean, the pollutants would spread quickly and an oil film would be formed in the sea. Lifetime of the oil film is decided by many factors, such as quantity, physical and chemical properties of oil, local air, sea power and geography, and it becomes thinner and thinner due to self-diffusion and effects of factors, such as wind, wave, tide, and stream. Furthermore, because of phys-chemical process, the films would evaporate, dissolve or emulsify.

Finally, the residue would solidify into solid particles and biological degradation would occur accordingly.

\section{Consequences}

\section{Effects on Photosynthesis}

By opaque oil film, light permeability would be reduced, water temperature would drop, O2$\mathrm{CO} 2$ balance in oceans would be destroyed, and algae's photosynthesis in contaminated water would be seriously influenced. What's worse, algae growth has significant effects on growth and reproduction of marine animals, resulting in destruction of entire marine ecosystem [8-9].

\section{Effects on Dissolved Oxygen in Seawater}

Lots of oxygen in water would be consumed by oil degradation, and the dominant way of seawater reoxygenation, namely, dissolution of atmosphere oxygen, is blocked by oil film, resulting in seawater hypoxia.

\section{Biological Productivity in Offshore Areas}

Most of crustaceans and marine fish produce in this region, so that marine living beings might be affected [10].

Effects of photo synthesis of algae in the ocean Oil pollution would reduce content of dissolved oxygen and destroy bio-physiological functions. Hence, the influence of marine fishery resources cannot be estimated in an accurate way. 


\section{Global Effects}

Considering CO2 exchange between ocean and atmosphere, green-house effect would go worse by oil pollution. What's more, EI Nino phenomenon would happen more frequently, resulting in intensification of "global problem"[11].

\section{Destruction of Coastal Wetlands}

Tanker oil spill and oil wells injection can be found both on- and off-shore. What's worse, the contaminated water might quickly spread to coastal wetlands, and destroy ecosystem of coastal wetlands.

\section{Red Tides}

In the serious oil-polluted marine area, the occurrence possibility of red tides would increase, and the extreme weather would have negative effects on seawater.

\section{Effects of Tourism}

The offshore oil can easily stick to shores, and beach entertainment would be contaminated accordingly. Hence, tourism development in the city would be influenced.

\section{Control Measures}

Much more attention has been drawn to offshore oil exploration. It should be noticed that with marine energy exploitation, the ocean would be polluted and ecological balance of marine biological diversity would be destroyed. Hence, sustainable development should be insisted on and effective measures should be taken to minimize the impact of oil exploration and development on marine life.

\section{Management Measure}

With rapid economic development, some of China's relevant laws and regulations, such as Protection of Marine Environment, should be guaranteed to be implemented, and modification and improvement can be made if necessary. What's more, more work can be done to improve supervision system of marine environment protection and enforcement mechanism. Meanwhile, law enforcement should be ensured to strengthen the protection of marine environment, to enforce the laws and regulations, and to fight against all illegal actions about environmental pollution to keep marine lives from destruction of offshore oil exploration [12].

It is necessary to strengthen environmental awareness of workers in exploration of offshore oil and to highlight staff training about environmental protection knowledge and skills.

In addition, universal participation should be encouraged in ocean protection to change people's concept driven by interests and to prevent marine ecological environment from destruction. It is notable that the damage to marine environment is long lasting, instead of temporary.

As for monitoring and early warning, information technology and real-time dynamic monitoring should be fully made use of. In foreign countries, oil spill and toxic substances emergency system (MARINESPIL GIS), remote sensing and geographic information system based on emergency response system (AOS) and offshore waters monitoring system have been developed.

The apparent gap of offshore oil exploration and pollution prevention between China and developed countries should be fully aware of. Therefore, international cooperation and exchanges should be strengthened, and monitoring of marine oil pollution and early warning systems should be reinforced.

\section{Technical Measures}

According to international practice management and recycle techniques, treatment methods of oil pollution can be divided, as follows:

i) Physical treatment methods.

In detail, the polluted water can be cleaned by disposal ship and its accessory recycling equipment, oil boom, and oil-absorbing materials.

ii) Chemical treatment methods.

In detail, the pollutants can be burnt or treated with chemical treatment agent, such as emulsifying dispersant, condensate oil, set oil, and settlement agent.

iii) Biological treatment methods. 
Artificial selection and cultivation can be made use of to improve phagocytic oil microbes to advance artificial petroleum hydrocarbon biodegradation.

In general, biological degradation enjoys special advantages, such as high speed, no residue and low cost, which has been the focus of researches available. The rate of microbial degradation for petroleum hydrocarbons is closely related to type and quantity of microorganisms, temperature of medium, and dispersion degree. The higher the dispersing degree is the higher degradation rate would be.

\section{Further Suggestions}

\section{From the Ship Owner}

To solve these problems, education plays an important role in rectifying it. An enterprise as a social member has its social responsibilities to improve the quality of lives and operations of other enterprises and organizations, other than dedicates to single-minded drive for economic efficiency and the runaway pursuit of profit in its core business. Via education to urgent the enterprise to establish its enterprise culture which including the concepts of environment protection. Designing the education course, on the one hand, it is necessary to provide the result that pollution had been happened in some underdeveloped countries; on the other hand, there are some countries who do well work on environment protection, such as Norway, Sweden and Denmark; it is a good approach to visit the real scene in these countries, meanwhile, the valuable experiences can be obtained by communicating with relevant enterprises, organizations or government departments.

\section{For the Reason of Crew Members}

Crews' performance to prevent pollution from ships is extremely important. Firstly, some incompetent crews did not know how to use these pollution prevention equipments and devices. They were trained properly at before and passed the examination and assessment of operational practice. However, when they took part in their jobs, they forgot the redundant procedures or operated different types of equipments; thereby the violation of regulation appeared conveniently. Secondly, the crews know the procedures very well and operate the equipments correctly, but, they are reluctant to use these equipments. It is because the heavy work load, complex procedures, lack of environment protection conception, and laziness. Finally, it is the owners or companies who encourage the crew to violate the regulations [13].

To solve these above problems, above all, education can be introduced to strengthen the concept of environment protection for crews, just like the education courses for ship owners which make the crew "know why", and then they have the willingness to protect the environment. Besides, pertinent regulations should be introduced properly for relevant officers and engineers; furthermore, a qualification training includes fundamental knowledge not only necessary for the duties of that position but also satisfied for testing of individuals on the systems they will be required to operate or maintain, which is the key issue, the existing training model should be changed and the crews' working capability should be cultivated, which make the crews to have the capability to use the equipments and devices; moreover, the on-job training and the situation familiar training about the special ship is necessary to be carried out duly and bearing in mind that continuously training on knowledge updating is needed. By doing so which makes crews "know how".

\section{From the Aspects of Supervision}

The supervisions are one of the most important defenses on combating against marine environment pollution, which can be defined as to inspect the compliance with associated regulations of the ships' operation and conditions. Firstly, the capabilities of super-visors to identify the illegal operation are different, and the awareness of their responsibilities is various. Secondly, the existing of paying under table simulates the ships to pollute the environment with very low cost. Finally, the means of surveillance is too old to keep up with the development of shipping industry.

The purpose of surveillance is to function as a deter-rent for arbitrary discharging ship-generated wastes, as a means of detection of discharges already made, and as a tool to combat, as effectively as possible the spills that have been detected. 
To combat with the pollution, it is necessary to find out and continuously pursue the real situations of ship operations on its voyage. All the inspectors should know well the operation procedures and some potential problems in order to examine the practices of crews; in addition, the inspectors should keep alert to identify miner clue of incompliance with regulations. Furthermore, the surveillance method should be updated through adopting helicopter and satellite aids. Airborne surveillance which increases the ship's risk of being caught in the process of making illegal discharges can be an effective measure to prevent discharges and thus reduce marine pollution from shipping [14].

\section{Strengthen Legislation Approach}

Meanwhile, the policy of criminal exposure should be established widely to counter against illegal operation. As this aspect is concerned, when the award are attractive enough, people was encouraged to expose these illegal operations. The United States has done good examples; the whistle blowers. Award was authorized by the bounty provision in the act to prevent pollution from ships, which allows courts to award up to one-half of the criminal fine to those who provided information leading to the conviction. These experiences should be considered by every responsible government to counter against pollution and this method may be the right way to combat for illegal discharge of waste oil at seas which are more difficult to cope with via traditional means.

\section{Conclusions}

In spite of the shipping industry is related various pollution, the modern economy must depend on this industry to sustain our modern lives. What we can we do is to minimum the pollution from ships, under the help of IMO's conventions, and the key issue is to implement these regulations; operators are needed not only competency but also having the willingness to do that. Thereby, the education can improve the concepts of environment protection for both ship owners and seafarers. So far as the supervision concerned, the skilled officers and modern surveillance models are very important factors which help to identify the violation of regulation clues. In addition, the reporting of illegal operation is another defense to protect the marine environment by providing considerable reward to reporters to encourage the exposure and warn the company to forbid illegal operation.

In general, there need an education protocol for all the interest parties to encourage them have the willingness to protect the marine environment. Besides, designing legislation, the high level reward for illegal discharge reporter mechanism should be popularized by all governments.

\section{Acknowledgements}

This study is supported by the Natural Science Foundation of Zhejiang Province of China (Grant No. LY13E090004), and supported by the Natural Science Foundation of Zhejiang Province of China (Grant No. LY12E09004).

\section{References}

[1] CHEN Xin-en. Research of the Technology for Dealing with Shipping Waste[J], Journal of Guangzhou Maritime Institute, 2013, 21( 4):5-7.

[2] QU Liang. Analysis of the Pollution Prevention and Control of the Sea Oil[J], Ocean Development and Management, 2012,13(5):77-81.

[3] LAN Hai-yan. Analysis the Marine Environmental Pollution Prevention and Control[J]. Journal of Hebei Normal University, 2010, 33(1): 74-78.

[4] WU Hong-qu. The Effects on Offshore Oil Exploration and Exploitation to Marine Biological and Its Countermeasures of Prevention and Control[J]. China Water Transport, 2011, 11(3):131-132. [5] ZHOU Zhi-quan, KONG Fang. The Marine Ecological Environment Pollution and Prevention Countermeasures in China [J].Ecological Environment, 1998, 12(2): 15-17.

[6] CAO Wei, DAI Wei. Countermeasure about the Disposal of Solid Waste on Surface Warship[J], Ship Science and Technology, 2014, 36( 11):176-179. 
[7] DENG Yu-hai , SHOU Xu-ri. Analysis of Disposing Program of Ship Wastes[J], Environmental Protection in Transportation,2000,21( 4):36-38.

[8] MA Sen-shu. Legislation Issues of Combating Marine Environment Pollution from Ships[J], Chinese Journal of Maritime Law, 2013, 24( 2) :3-7.

[9] CHENG Chuan-liang,YAN Xun-tang. Ships to Sea Pollution and Government Measure[J], China Water Transport (Academic Version), 2006,6(7):12-13.

[10] CAI Chi-xue, JIAO Song-fei. China's Oil Pollution in Coastal Waters Situation and Control Measures[J]. Chemical Technology and Development, 2012, 41(8): 14-17.

[11] BIN Zhi-qi, YU Hui. Prevention and Control Measures for the Marine Oil Pollution in Fang cheng gang [J]. The Public Science and Technology, 2011, 23(4): 110-111.

[12] CHEN Yan. China's Offshore Oil Pollution Present Situation and Prevention[J]. Industrial Safety and Environmental Protection, 2003, 29(11): 20-24.

[13] FANG Xing, YANG Wei. Research and Prevention on Marine Oil Pollution[J]. Environmental Science and Management, 2007, 32(9): 78-80.

[14] NI Guo-chao, ZHAO Wu-feng. Influence and Control of Oil Pollution of the Marine Environment[J]. Production and the Environment, 2005, 5(12): 14-15. 